\title{
SOME REMARKS ON THE THEORY OF GRAPHS
}

\section{P. ERDÖS}

The present note consists of some remarks on graphs. A graph $G$ is a set of points some of which are connected by edges. We assume here that no two points are connected by more than one edge. The complementary graph $G^{\prime}$ of $G$ has the same vertices as $G$ and two points are connected in $G^{\prime}$ if and only if they are not connected in $G$.

A special case of a theorem of Ramsey can be stated in graph theoretic language as follows:

There exists a function $f(k, l)$ of positive integers $k, l$ with the following property. Let there be given a graph $G$ of $n \geqq f(k, l)$ vertices. Then either $G$ contains a complete graph of order $k$, or $G^{\prime}$ a complete graph of order $l$. (A complete graph is a graph any two vertices of which are connected. The order of a complete graph is the number of its vertices.)

It would be desirable to have a formula for $f(k, l)$. This at present we can not do. We have however the following estimates:

TheOREM I. Let $k \geqq 3$. Then

$$
2^{k / 2}<f(k, k) \leqq C_{2 k-2, k-1}<4^{k-1} .
$$

The second inequality of Theorem I was proved by Szekeres, ${ }^{1}$ thus we only consider the first one. Let $N \leqq 2^{k / 2}$. Clearly the number of different graphs of $N$ vertices equals $2^{N(N-1) / 2}$. (We consider the vertices of the graph as distinguishable.) The number of different graphs containing a given complete graph of order $k$ is clearly $2^{N(N-1) / 2} / 2^{k(k-1) / 2}$. Thus the number of graphs of $N \leqq 2^{k / 2}$ vertices containing a complete graph of order $k$ is less than

$$
C_{N, k} \frac{2^{N(N-1) / 2}}{2^{k(k-1) / 2}}<\frac{N^{k}}{k !} \frac{2^{N(N-1) / 2}}{2^{k(k-1) / 2}}<\frac{2^{N(N-1) / 2}}{2}
$$

since by a simple calculation for $N \leqq 2^{k / 2}$ and $k \geqq 3$

$$
2 N^{k}<k ! 2^{k(k-1) / 2} \text {. }
$$

But it follows immediately from (1) that there exists a graph such that neither it nor its complementary graph contains a complete subgraph of order $k$, which completes the proof of Theorem I.

The following formulation of Theorem I might be of some interest:

Received by the editors April 30, 1946, and, in revised form, October 20, 1946.

${ }^{1}$ P. Erdös and G. Szekeres, Compositio Math. vol. 2 (1935) pp. 463-470. 
Define $A(n)$ as the greatest integer such that given any graph $G$ of $n$ vertices, either it or its complementary graph contains a complete subgraph of order $A(n)$. Then for $A(n) \geqq 3$

$$
\frac{\log n}{2 \log 2}<A(n)<\frac{2 \log n}{\log 2} .
$$

The proof of (2) follows immediately from Theorem I. $\left(4^{\Lambda(n)}>n\right.$, $2^{4(n) / 2}<n$.)

The general theorem of Ramsey will now be stated.

TheOREM (RAMSEx). Given three positive integers $i, k, l, i \leqq k, i \leqq l$, there exists a function $f(i, k, l)$ with the following property: If $n \geqq f(i, k, l)$ and if there is given a collection of combinations of order $i$ of $n$ elements, such that every combination of order $k$ contains at least one combination of order $i$ of the collection, then there exists a combination of order $l$ all of whose combinations of order $i$ belong to the collection.

Several proofs of this theorem have been published. ${ }^{1}$ Szekeres's proof gives the best known limits for $f(i, k, l)$. He proves ${ }^{1}$

$$
f(i, k, l) \leqq f(i-1, f(i, k-1, l), f(i, k, l-1))+1 ;
$$

also clearly $f(1, k, l)=k+l-1 ; f(i, i, l)=l ; f(i, k, i)=k$. By the same method we used in the proof of Theorem I we obtain that for sufficiently large $k$

$$
f(i, k, k)>c^{k^{i-1} / i 1}
$$

or

$$
A(i, n)<c_{1}(\log n)^{1 /(i-1)} .
$$

(To see this put $k=c_{1}(\log n)^{1 /(i-1)}$ in (4) and observe $f(i, k, k)>n$, for sufficiently large $c_{1}$.) Here $c$ and $c_{1}$ depend only on $n$ and $i$, and $A(i, n)$ is the greatest integer with the following property: Split the combinations of order $k$ of $n$ elements into two classes $U_{1}$ and $U_{2}$ in an arbitrary way. Then there exist $A(i, n)$ elements all whose combinations of order $k$ are either in $U_{1}$ or in $U_{2}$. The values given by (4) are very much smaller than the values given by (3).

From (3) we obtain ${ }^{1}$

$$
f(k, l)=f(2, k, l) \leqq C_{k+l-2, k} .
$$

Thus

$$
f(3, l) \leqq C_{l+1,2} .
$$

It is possible that 


$$
f(3, l)=O(l) .
$$

Our method used in the proof of Theorem I does not enable us to show that $f(3, l) / l \rightarrow \infty$.

Before concluding we prove the following theorem.

THEOREM II. Let there be given $(k-1)(l-1)+1$ integers $a_{1}<a_{2}$ $<\ldots$. Then either there exist $k$ of them no one dividing the other or $l$ of them each a multiple of the previous one.

Construct a matrix $a_{i}^{(j)}$ with the following properties: (1) no $a_{i}^{(j)}$ is a multiple of any $a_{i}^{(r)}$ with $j \leqq r$; (2) every $a_{i}^{(r+1)}$ is a multiple of some $a_{t}^{(r)}$; (3) all the $a$ 's occur among the $a_{i}^{j}$ once and only once. If any row contains $k$ or more elements we have $k a$ 's, no one dividing the other. If not, it clearly follows that the number of rows must be at least $l$. Now we obtain from (2) that by considering any $a_{i}^{(l)}$ we obtain a sequence of $l a$ 's, each being a multiple of the previous one, which completes the proof. The $(k-1)(l-1)$ integers $p_{u}^{0}, 1 \leqq u \leqq k-1$; $1 \leqq v \leqq l-1, p_{u}$ primes, show that $(k-1)(l-1)+1$ is best possible. ${ }^{2}$

By the same method we can prove the following theorem.

THEOREM IIa. Let there be given a graph $G$ of $(k-1)(l-1)+1$ vertices. Then either $G$ contains a complete graph of order $k$, or $G^{\prime}$ contains a directed path of $l$ vertices, for every orientation of the edges of $G^{\prime}$ in which there are no directed closed paths.

Recently very much more general theorems have been proved by Grünwald and Milgram. ${ }^{3}$ They in fact proved (among others) that the condition that $G^{\prime}$ contains no closed directed path is superfluous.

We suppress the proof of Theorem IIa since it is essentially the same as that of Theorem II. (We only remark that $a$ connected to $b$ by a line directed from $a$ to $b$ should be replaced by $a$ divides $b$.)

\section{STANFORD UNIVERSITY}

${ }^{2}$ This proof is due to $\mathrm{J}$. Brunings.

${ }^{3}$ Oral communication. 